\title{
From Uruk to Ur: Automated Matching of Virtual Tablet Fragments
}

Gehlken, Erlend (Universität Frankfurt), Tim Collins (University of Birmingham), Sandra Woolley (University of Birmingham), Laurence Hanes (University of Birmingham), Andrew Lewis (University of Birmingham), Luis Hernandez Munoz (University of Birmingham), and Eugene Ch'ng (University of Nottingham)

Unlike jigsaw puzzles of thousands of pieces, which computers can now solve, fragmented tablets constitute much more complex freeform three-dimensional "puzzles" whose pieces can belong to an unknown number of complete or incomplete tablets. Computer-aided reconstruction of archaeological fragments has been an active area of research in recent years, although most published work has been specific to the joining of potsherds. Challenges in automated reassembly, aside from the difficulties of acquiring three dimensional scans, include the extremely difficult search problems, the large numbers of false-positive matches and the incorporation of surface imagery with object geometry.

We have produced and refined a means of automatically joining fragments. The process of automated computer joining comprises three main stages: preprocessing, pairwise matching and match ranking. Pairwise matching is an enormously computationally intensive problem that significantly benefits from the parallel computing capacity of modern graphical processing units.

The first successful pair of automated fragment joins was achieved in 2014 for a pair of fragments from Uruk. Further automated joins have since been made using fragments from Ur, currently held by the British Museum. The 3D virtual Ur fragments were acquired with high-resolution photographic texture using a novel, portable, low-cost "scannerless" rotary photogrammetric scanning system. The workflow for the method was designed such that minimal user intervention or training is required for both the 3D acquisition and the automated joining. Future ambitions include the achievement of long-distance photogrammetric matches, for example, of London and Pennsylvania virtual tablet fragments. 